\title{
Spatio-temporal Descriptor Using 3D Curvature Scale Space
}

\author{
A. Dyana and Sukhendu Das \\ Visualization and Perception Lab, Computer Science and Engineering Deptt. \\ Indian Institute of Technology Madras, Chennai, India \\ dyana@cse.iitm.ernet.in, sdas@itm.ac.in
}

\begin{abstract}
This paper presents a novel technique to jointly represent the shape and motion of video objects for the purpose of content based video retrieval (CBVR). It enables to retrieve similar objects undergoing similar motion patterns, that are not captured only using motion trajectory or shape descriptors. In our approach, both shape and motion information are integrated in a unified spatio-temporal representation. Curvature scale space theory proposed by Mokhtarian is extended (in 3D) to represent shape as well as motion trajectory of video objects. A sequence of $2 \mathrm{D}$ contours are taken as input and convolved with a $2 \mathrm{D}$ Gaussian. The zero crossings are found out from the curvature of evolved surfaces, which form the 3D CSS surface. The peaks from the 3D CSS surface form the features for joint spatio-temporal representation of video objects. Experiments are carried out on CBVR and results show good performance of the algorithm.
\end{abstract}

Keywords: Curvature scale space, motion trajectory, content based video retrieval, spatio-temporal descriptor.

\section{Introduction}

As there is an increase in availability of video data, there is a need to describe video based on its content. MPEG-4 provides access and manipulation of video objects and MPEG-7 describes the features of the multimedia content. Shape and motion are important features in content based video retrieval. Shape descriptors (spatial domain) are classified into two categories: region based and contour based. A contour-based descriptor encapsulates the shape properties of the object's outline (silhouette). Fourier descriptors, Medial axis transform, Shape signature [1, Shape context [2, Geometric representations, Grid representation [3] are some of the contour based techniques to represent object shapes.

Multiscale description of contours is an emerging research area for describing shapes. The Multiscale Fourier descriptors improves the shape retrieval accuracy of the commonly used Fourier descriptors [4. A multiscale extension to the medial axis transform (MAT) [5] or skeleton is obtained by combining information derived from a scale-space hierarchy of boundary representations with region information provided by the MAT. Two leading approaches emerged: one by Latecki et al. 6] based on the best possible correspondence of visual parts 
and a second approach developed by Mokhtarian et al. based on the curvature scale space (CSS) representation [7. Both descriptors are based on the computation of a similarity measure on the best possible correspondence between maximal convex/concave arcs contained in simplified versions of boundary contours. It is impossible to use the CSS descriptor to distinguish between totally convex shapes. Given the above observations, a new shape description method termed multi-scale convexity concavity (MCC) representation was proposed by T. Adamek [8]. A multiscale, morphological method for the purpose of shapebased object recognition was proposed 9 .

Motion trajectory describes the displacements of objects in time, where objects are defined as spatio-temporal regions. For modeling object's trajectory, Dimitrova and Golshani [10 utilized a chain coding scheme to represent different objects' movements. Little and Gu [11] used an interpolation scheme to model object trajectories by using a set of polynomial basis. In addition, Sahouria [12] applied a Harr transform for representing object trajectories in spatial domain through a multiscale analysis. In addition, Dagtas et al. 13] proposed a trajectory-based model and a trail-based model for video retrieval by taking advantages of the Fourier transform and Mellin transform, respectively. Two affine-invariant representations for motion trajectories based on curvature scale space (CSS) and centroid distance function (CDF) was derived.

Spatio-temporal descriptors to represent position and motion of regions has been proposed in 14 15. The position, motion and color describes the region in a high dimensional space. $\mathrm{K}$ nearest neighbor retrieval was used in 14. In [15], a Graph based description was used to describe relation between regions. In content based video retrieval systems such as Netra-V [16, the shape and motion features are represented individually and the match results are integrated to select a video clip. Chang et al. 17] proposed a VideoQ system for video searching using a set of visual features like color, texture, shape, and motions. In our proposed method, the standard 2D CSS representation is modified to represent both shape and motion of video objects in a unified spatio-temporal representation. The deformed shapes over time are taken as input and a 3D CSS descriptor is generated for each video object.

\section{Standard Curvature Scale Space}

A CSS image can be considered as a multi-scale organization of the invariant local features of a free-form 2D contour. The CSS image is a multi-scale organization of the inflection points (or curvature zero-crossing points) of the contour as it evolves. Curvature is a local measure of how fast a planar contour is bending. Contour evolution is achieved by first parameterizing by arc length. This involves sampling the contour at equal intervals and recording the $2 \mathrm{D}$ coordinates of each sampled point. The result is a set of two coordinate functions (of arclength) which are then convolved with a 1D Gaussian filter of increasing width or standard 
deviation. Curvatures are computed for all the smoothed contours. As a result, curvature zero-crossing points can be recovered and mapped to the CSS image in which the horizontal axis represents the arclength parameter $(\mathrm{u})$ on the original contour, and the vertical axis represents the standard deviation (scale) of the Gaussian filter. Fig. 1 illustrates an example for 2D CSS representation. Fig. 10 is the CSS image for the fish contour shown in Fig. 17. The maximas of lower scale are considered to be noise which is ignored in the matching stage. The features recovered from a CSS image for matching are the maxima of its

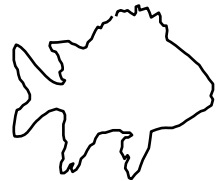

(a)

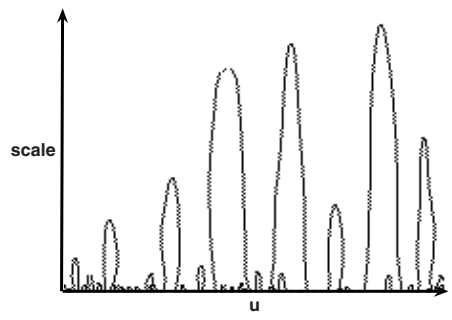

(b)

Fig. 1. 2D CSS image (b) for the fish shape in (a)

zero-crossing contours [18. The matching of two CSS images consists of finding the optimal horizontal shift of the maxima in one of the CSS images that would yield the best possible overlap with the maxima of the other CSS image. The matching cost is then defined as the sum of pairwise distances (in CSS) between corresponding pairs of maxima.

\section{Proposed 3D CSS Representation}

The curvature scale space for 2D contours proposed by Mokhtarian [19] for planar curves, which has been standardized in MPEG-7, is extended in our work. This is a joint spatio-temporal representation of moving objects in a video (shot) and is similar to the 3D surface representation in [7].

Mokhtarian represented 2D contour by a parametric vector,

$$
r(u)=((x(u), y(u))
$$

We represent the input sequence of contours by the following equation which has two parameters: $\operatorname{spatial}(\mathrm{u})$ and temporal (v), as

$$
r(u, v)=((x(u, v), y(u, v))
$$

To normalize the arc length $(\mathrm{u})$, the contour is sampled and represented by 200 equally spaced points. Similarly, the temporal parameter (v) is normalized by 
uniformly sampling the trajectory and representing by 40 points. The formula for computing the curvature is extended from $2 \mathrm{D}$ as:

$$
\kappa(u, v)=\frac{\dot{x}(u, v) \ddot{y}(u, v)-\dot{y}(u, v) \ddot{x}(u, v)}{\left(\dot{x}(u, v)^{2}+\dot{y}(u, v)^{2}\right)^{3 / 2}}
$$

If,

$$
\Gamma=\{(x(u, v), y(u, v)) \mid u \in[0,1], v \in[0,1]\}
$$

then the evolved contours when convolved with Gaussian is given by

$$
\Gamma_{\sigma}=\left\{\left(X_{\sigma}(u, v), Y_{\sigma}(u, v)\right) \mid u \in[0,1], v \in[0,1]\right\}
$$

where

$$
\begin{gathered}
X_{\sigma}(u, v)=x(u, v) \otimes g_{\sigma}(u, v) \\
Y_{\sigma}(u, v)=y(u, v) \otimes g_{\sigma}(u, v) \\
g_{\sigma}=\frac{1}{2 \pi \sigma^{2}} e^{-u^{2}+v^{2} / 2 \sigma^{2}} \quad \text { is a } 2 \mathrm{D} \text { Gaussian function. } \\
\text { and } \otimes \text { indicates the 2D convolution function. }
\end{gathered}
$$

Using Eqns. 3 and 5, the curvature of the evolved contours is given by,

$$
\kappa_{\sigma}(u, v)=\frac{\dot{X}_{\sigma}(u, v) \ddot{Y}_{\sigma}(u, v)-\dot{Y}_{\sigma}(u, v) \ddot{X}_{\sigma}(u, v)}{\left(\dot{X}_{\sigma}(u, v)^{2}+\dot{Y}_{\sigma}(u, v)^{2}\right)^{3 / 2}}
$$

where

$$
\begin{array}{r}
\dot{X}_{\sigma}(u, v)=\frac{\partial}{\partial u}\left(x(u, v) \otimes g_{\sigma}(u, v)\right)+\frac{\partial}{\partial v}\left(x(u, v) \otimes g_{\sigma}(u, v)\right) \\
=x(u, v) \otimes \dot{g_{\sigma}}(u, v)
\end{array}
$$

and

$$
\begin{array}{r}
\ddot{X}_{\sigma}(u, v)=\frac{\partial^{2}}{\partial u^{2}}\left(x(u, v) \otimes g_{\sigma}(u, v)\right)+\frac{\partial^{2}}{\partial v^{2}}\left(x(u, v) \otimes g_{\sigma}(u, v)\right) \\
=x(u, v) \otimes \ddot{g}_{\sigma}(u, v)
\end{array}
$$

Similarly,

$$
\begin{aligned}
& \dot{Y}_{\sigma}(u, v)=y(u, v) \otimes \dot{g}_{\sigma}(u, v) \\
& \ddot{Y}_{\sigma}(u, v)=y(u, v) \otimes \ddot{g}_{\sigma}(u, v)
\end{aligned}
$$

The symbol $\otimes$ used in Eqns. 8 - 10 indicates a 2D convolution operation. To implement the same, the numerical addition of two separable derivatives of the gaussian function $(\mathrm{g})$ along $\mathrm{x}$ and $\mathrm{y}$ is taken, and then convolved with the $2 \mathrm{D}$ function (x or $\mathrm{y})$. 


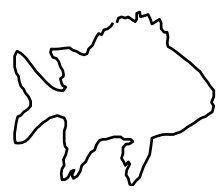

(a)
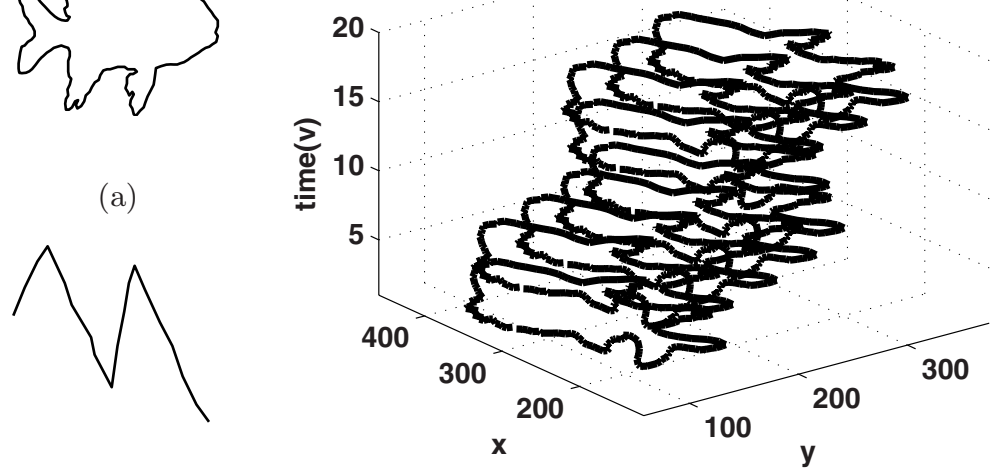

(b)

(c)

Fig. 2. Input sequence of contours in (c) for the object (a) fish and its trajctory in (b)

The solution of the equation given in 7

$$
\kappa_{\sigma}(u, v)=0
$$

gives the $2 \mathrm{D}$ zero crossing contours.

Fig. 2 shows the input sequence of contours of a fish shown in Fig. 2 a which is translated along the trajectory shown in Fig. $2 \mathrm{~b}$. The contour of the fish is represented by $\mathrm{x}$ and $\mathrm{y}$ coordinates and the fish is moved along the trajectory over time. The change in the contour position is depicted along the $\mathrm{v}$ axis. The surface thus formed by the stack of 2D contours, is evolved by a 2D Gaussian function at different scales $(\sigma)$. The surface can be interpreted as a set of normalized 2D contours, where the $\mathrm{z}$ coordinate is $\mathrm{v}$. Hence the curvature calculation can be extended from 2D as shown in Eqn. 3. As the contours get evolved it is smoothed in both the dimensions, spatial and temporal. The surface eventually transform into a cylindrical structure. When viewed along the spatial coordinates ( $\mathrm{x}$ and y), the contour evolves into an elliptical structure.

\section{3D CSS Surface}

3D CSS surface is a 3D plot of the zero crossings at each level. Axis variables are the spatial parameter $(\mathrm{u})$, the temporal parameter $(\mathrm{v})$ and $\operatorname{scale}(\sigma)$. The surface (see Fig. 3) consists of a series of hills and valleys. Every hill corresponds to the successive pair of occurances of convexity and concavity (or vice-versa) in the sequence of contours (i.e surface). The local peaks are found out by first deleting the global peak at the largest value of sigma, and then recursively traversing down and eliminating its neighbours until a valley is reached. The set of peaks 


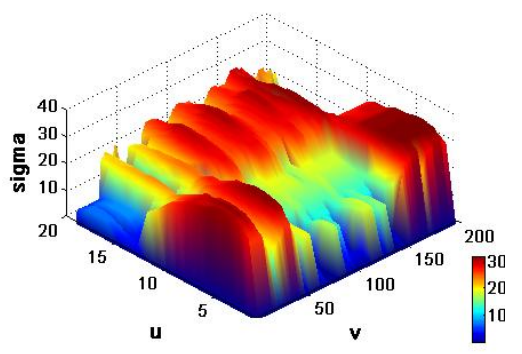

Fig. 3. 3D CSS surface

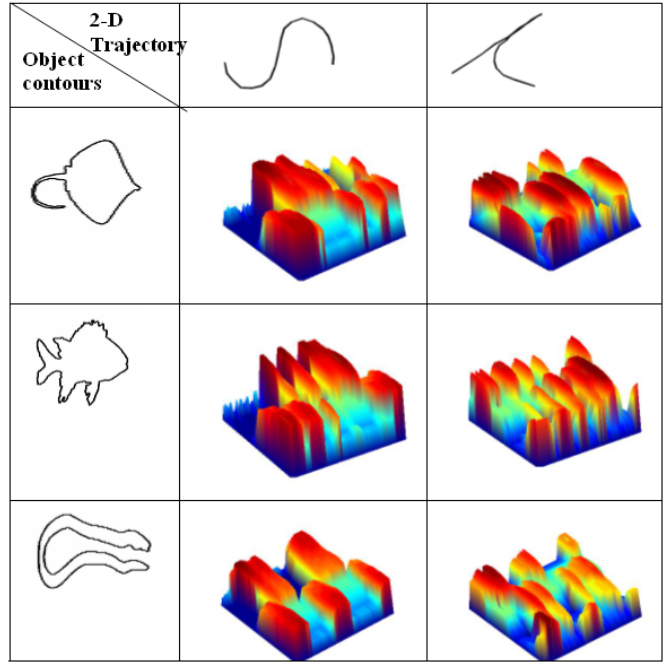

Fig. 4. 3D CSS surfaces for three different objects and two different motion trajectories

form the features to jointly represent shape and motion of the object. The overall algorithm of the proposed method is given in the following section.

\subsection{Overall Algorithm}

Input for the system is a sequence of $2 \mathrm{D}$ contours of video objects. From a set of consecutive frames, contours are taken and stacked over time to form a 3D surface. $\mathrm{x}$ and $\mathrm{y}$ axis is characterized by spatial parameter $(\mathrm{u})$ and $\mathrm{z}$ axis by a temporal parameter (v) (see Eqn. 2). The steps of the overall algorithm are as follows:

1. The $3 \mathrm{D}$ surface is smoothed by a $2 \mathrm{D}$ Gaussian of minimum $\sigma$ to obtain an evolved surface (Eqn. 5)

2. Curvature of the evolved surface is computed using Eqn. 3

3. The zero crossings of the curvature in the spatial as well as temporal dimensions are found out and the locations $(u, v, \sigma)$ of the same are stored.

4. Increment the value of sigma and repeat steps 2 and 3.

5. The set of zero crossings are marked for each sigma which forms a 3D CSS surface as shown in Fig. 3 ,

6. The peaks from the 3D CSS surfaces are obtained which form the features for the moving object.

7. To match the query with the models from the database the peaks of the query and model are matched by the matching algorithm described in section 4.2

Fig. 4 illustrates the discriminating ability of the 3D CSS representation to distinguish between different combinations of trajectories and objects. A contour 
of a fish is taken and it is moved or translated along the trajectory to obtain a 3D CSS surface. Three different shape contours along two different trajectories are shown. For the same trajectory (motion) and for different objects, the 3D CSS surfaces have similar structure along v axis (the time axis). For the same object with different motions (trajectories), the 3D CSS surfaces have similar structure along $\mathrm{u}$ axis (the parameter for object contours). As observed from the figure, different combinations of trajectories and objects provide different structures of the 3D CSS surface. This shows the uniqueness property of the algorithm to represent the spatio-temporal information of an object shape in a video.

\subsection{D CSS Matching Algorithm}

Matching algorithm proposed for matching CSS maxima in 2D space by Mokhtarian has been extended for matching the set of peaks on a 3D surface in our case. Every video object in the database is represented by the locations of peaks $(u, v, \sigma)$ in its 3D CSS surface. The location of peaks are normalized. The set of peaks which form the features representing the shape trajectory pair for the query is matched with the set of peaks in the database. The pairs are retrieved according to the match-cost. The lower the match-cost, higher is the similarity of the model with the query.

In the standard CSS, the maximas $(u, \sigma)$ in the CSS image form the features. In our 3D CSS, the location of the peaks represented by triplets $(u, v, \sigma)$ form the features. The feature list is sorted by the scale coordinate of the peaks. The highest scale maximum of the query is matched with the model whose $\sigma$ coordinate lies close (within 80\%) to that of the highest model peak. For each matched peak, nodes are created with two lists. The first list contains the peaks of the query, the second list contains its corresponding matching peak in the model. The algorithm proceeds in similar to the standard 2D CSS matching algorithm. In the standard 2D CSS matching algorithm, the horizontal distance of the maxima and the height of the peaks contribute to the match-cost. In our algorithm, the euclidean distance (in 2D space) between the peaks and the height of the peaks contribute to the match-cost.

The system has the property of translation, rotation and scaling invariants of shape and trajectories. This is based on the principle that 2D CSS is also invariant to rotation, translation and scaling. It works based on the principle that similar video objects should have similar representations.

\section{$5 \quad$ Experimental Results}

Objects used for our experiments are from MPEG7-B dataset 20] and a part of the SQUID database [18 (marine animals). The trajectory set is part of the database downloaded from 21. For each combination of object and trajectory, a 3D CSS descriptor is generated and stored. For a query, the 3D CSS descriptor is obtained and matched with the models from the database. 


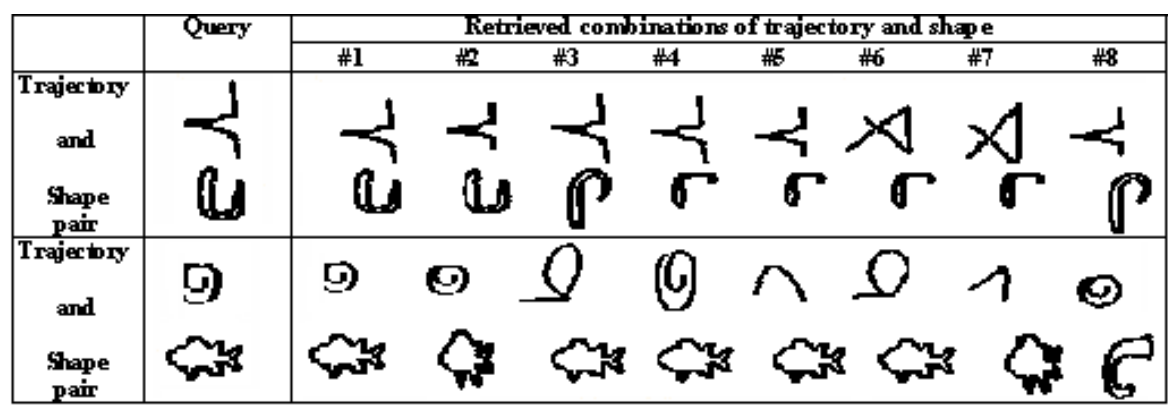

Fig. 5. Top eight shape and trajectory pairs retrieved for the query in the first row of the table, arranged in descending order of similarity

Table 1. Average Accuracy for retrieval estimated over first 10 outputs

\begin{tabular}{lc}
\hline Dataset & Accuracy (in percentage) \\
\hline MPEG7-B [20 & 74.78 \\
SQUID [18 & 77.01 \\
\hline
\end{tabular}

One set of experiments were conducted on 100 shapes from MPEG7-B dataset and 24 trajectories from 21. Other set of experiments involved 24 shapes of "fish" from SQUID database and 36 trajectories from [21]. Fig. 5] shows the results of retrieval for the query "fish" from SQUID, as displayed in the first column. All other entries (columnwise) show the shape and motion pairs retrieved, which are arranged in decreasing order of similarity (ie. ascending order of match-cost) from left to right. From the results, it is observed that, the retrieved pairs are similar to the query. The results show that the proposed method is invariant to translation, rotation and scale for both shape and trajectory. Perspective foreshortening due to sidewise movement of the marine animal, is not considered within the current scope of work. Table. 1 shows the average accuracy of retrieval estimated over 100 queries. Accuracy is calculated as the ratio of the number of correct outputs observed for the first 10 retrieved videos.

\section{Conclusion}

We have proposed a unique spatio-temporal 3D CSS representation to represent shape and motion of video objects. The deformation of shape is captured in the representation. The algorithm has shown good results for CBVR application. The 3D CSS matching algorithm can be modified to be more computationally efficient. 


\section{References}

1. Davies, E.R.: Machine Vision: Theory, Algorithms, Practicalities. Morgan Kaufmann, San Francisco (2005)

2. Belongie, S., J.M., Puzicha, J.: Matching shapes. In: Eighth IEEE International Conference on Computer Vision, pp. 456-461 (2001)

3. Lu,, Sajjanhar, A.: Region-based shape representation and similarity measure suitable for content-based image retrieval. Multimedia System 7(2), 165-174 (1999)

4. Kunttu, I., Lepistö, L., Rauhamaa, J., Visa, A.: Multiscale fourier descriptor for shape-based image retrieval. In: Proceedings of the 17th International Conference on Pattern Recognition (ICPR 2004), vol. 2, pp. 765-768.

5. Ogniewicz, L.R.: Skeleton-space: a multiscale shape description combining region and boundary information. In: Proc. Comput. Vision Pattern Recogn.

6. Latecki, L.J., Lakamper, R., Eckhardt, U.: Shape descriptors for non-rigid shapes with a single closed contour. In: CVPR, pp. 424-429

7. Mokhtarian, F., Bober, M.: Curvature Scale Space Representation: Theory, Applications and MPEG-7 Standardization. Kluwer Academic Publishers, The Netherlands (2003)

8. Adamek, T., O'Connor, N.E.: A multiscale representation method for nonrigid shapes with a single closed contour. IEEE Transactions on Ciruits and systems for video technology 14(5), 742-753 (2004)

9. Jalba, A.C., Wilkinson, M.H.F., Roerdink, J.B.T.M.: Shape representation and recognition through morphological curvature scale spaces. IEEE Transactions on Image Processing 15(2), 331-341 (2006)

10. Dimitrova, N., Golshani, F.: Motion recovery for video content classification. ACM Trans. Inf. Syst. 13(14), 408-439 (1995)

11. Little, J.J., Gu, Z.: Video retrieval by spatial and temporal structure of trajectories. In: Proc. SPIE Storage and Retrieval for Media Databases, vol. 13, pp. 408-439 (1995)

12. Sahouria, E.: Video indexing based on object motion. Master's thesis, Dept. Elect. Eng. Comp. Sci, Univ. California, Berkeley (1997)

13. Dagtas, S., et al.: Models for motion-based video indexing and retrieval. IEEE Transactions on Image Processing 9(1), 88-101 (2000)

14. DeMenthon, D., Doermann, D.: Video retrieval using spatio-temporal descriptors. In: ACM Multimedia, pp. 508-517 (2003)

15. Chatzis, S., Doulamis, A., Kosmopoulos, D., Varvarigou, T.: Video representation and retrieval using spatio-temporal descriptors and region relations. In: ICANN (2), pp. 94-103 (2006)

16. Deng, Y., Manjunath, B.S.: Netra-v: Toward an object-based video representation. IEEE Transactions on Ciruits and systems for video technology 8(5), 116-127 (1998)

17. Chang, S.F., et al.: A fully automated content-based video search engine supporting spatiotemporal queries. IEEE Transactions on Ciruits and systems for video technology 8(5), 602-615 (1998)

18. SQUID, http://www.ee.surrey.ac.uk/research/vssp/imagedb

19. Mokhtarian, F., Abbasi, S., Kittler, J.: Robust and efficient shape indexing through curvature scale space. In: British Machine Vision Conference, pp. 53-62 (1996)

20. MPEG7-BDataset, http://csr.bu.edu/asl/data/experiments/cikm2005/database2/

21. TrajectoryDatabase, http://mmplab.eed.yzu.edu.tw/trajectory/trajectory.rar 